1

Possui graduação em Engenharia Civil pela Universidade Federal do Rio Grande do Norte (2008) e especialização em Gestão e tecnologia na construção civil. Tem experiência na área de Engenharia Civil, com ênfase em Gestão, Planejamento e Orçamentos da Construção Civil. IFC - Instituto Federal Catarinense 2

Doutor em Engenharia Elétrica - USP - atuante no Centro Universitário UNISOCIESC, departamento de Engenharia de Produção, Joinville / SC - Brasil. Bacharel em Informática pela Universidade do Vale do Rio dos Sinos (1995), mestre em Ciências da Computação pela Universidade Federal de Santa Catarina (2001) 3

Mestre em Engenharia de Produção - UNISOCIESC alvaro.graziani@sociesc.com. br, atuante no Centro Universitário UNISOCIESC, departamento de Engenharia de Produção, Joinville / SC - Brasil.

\section{ELABORAÇÃO DE INDICADORES CHAVE DE DESEMPENHO: SETOR DE OBRAS PÚBLICAS DAS AUTARQUIAS FEDERAIS}

\author{
ELABORATION OF KEY PERFORMANCE INDICATORS: SECTOR \\ OF PUBLIC WORKS OF THE FEDERAL AUTHORITIES
}

Rommel Souza Silva ${ }^{1}$

Mehran Misaghi ${ }^{2}$

Álvaro Paz Graziani ${ }^{3}$

RESUMO: Este artigo define a gestão por resultados e os indicadores chave de desempenho (KPI) como técnicas gerenciais importantes para o setor de obras públicas das autarquias federais. A problemática identificada na pesquisa são os aditivos de custo e prazo às obras. A pesquisa classifica-se como pesquisa de natureza aplicada, com objetivos exploratórios, descritiva e com revisão bibliográfica. Os procedimentos utilizados estão classificados ora em estudo de campo, ora em estudo de caso, a abordagem quantitativa inclui os dados pertinentes aos contratos das obras públicas nas autarquias, que tem como apoio o Sistema Integrado de Monitoramento Execução e Controle (SIMEC). A amostra da população foi gerada pelas obras da Universidade e Institutos Federais de Santa Catarina, com total de 38 obras espalhadas em 17 campi. Por conseguinte, os dados foram analisados com correlação de Pearson ( $r$ ) e Data Envelopment Analysis (DEA). A pesquisa identificou as instituições com as obras mais eficiente em que a modalidade de licitação o Regime Diferenciado de Contratação (RDC), efetuou-se como benchmark para o setor, ainda apresenta um quadro com as dimensões, subdimensões e métricas a serem utilizadas pela gestão pública na indicação de resultados do setor de obras, ademais enquadrou as metas a serem atingidas pelas instituições.

PAlaVras-Chave: Indicadores chave de desempenho; Gestão por resultados; Benchmark; Obras públicas; Instituições Federais de Ensino.

AbSTRACT: This article defines the management by results and key performance indicators (KPI) as important managerial techniques for the public works sector of the federal authorities. The problems identified in the research are the cost and term additives for the works. The research is classified as a research of applied nature, with exploratory, descriptive and bibliographic review objectives. The procedures used are classified as a field study, or in a case study, the quantitative approach includes data pertinent to public works contracts in the municipalities, which is supported by the Integrated Monitoring and Execution and Control System (SIMEC). The sample of the population was generated by the works of the University and Federal Institutes of Santa Catarina, with a total of 38 works spread out on 17 campuses. Therefore, the data were analyzed with Pearson (r) correlation and Data Envelopment Analysis (DEA). The research identified the institutions with the most efficient works in which the modality of bidding the Differential Regime of Contracting (RDC), was made as benchmark for the sector, still presents a table with the dimensions, subdimensions and metrics to be used by the management public in the indication of results of the works sector, in addition it set the goals to be reached by the institutions.

KEYWORDS: Key performance indicators; Management by results; Benchmark; Public works; Federal Institutions of Education. 


\section{INTRODUÇÃO}

As Instituições Federais de Ensino (IFES) são organizações complexas que acompanha e fiscaliza a execução de obras públicas para Administração Pública Federal (APF) por meio do Setor de Obras Públicas (SOP), porém com a nova gestão pública que requisita indicadores formados por métricas para avaliar a situação particular do setor, vistos como técnica gerencial que faz uma mudança da abordagem na produção ou orientação para o custo ou para os resultados do setor de obras.

No setor público, a gestão corresponde em gerir recursos e bens, o que torna razoável a distribuição de recursos de que se dispõe, visando obter melhores resultados da gestão. Assim, identificados os aditivos de custo e prazo em acréscimo como a problemática, e que os indicadores servirão como base para a responsabilidade dos gestores pelo desempenho institucional, configurando-se como um dos principais objetivos do conceito de gestão por resultados que vem ao encontro do sucesso de obras públicas. Porém, demanda conhecimento da legislação e tomada de decisões por parte dos gestores, que vêm em busca de instrumentos para viabilizar o cumprimento dos interesses da APF e, para tanto, promova a realização de obras e serviços de manutenção a contento (GRIN, 2000; SORTE 2015).

Este artigo tem por objetivo elaborar indicadores chave de desempenho por meio de sistemas gerenciais para o setor de obras públicas e privadas, que possa ser usado para mensurar e comparar o desempenho de autarquias, precisamente as IFES, ou ser considerado como base para benchmarking, é um fator a ser desenvolvido, para a gestão que busca resultados de sucesso.

\section{REFERENCIAL TEÓRICO}

A introdução do New Public Management (NPM), descrita por Fonseca et al. (2013), traduzida como Nova Gestão Pública, no Brasil na década de 1990, caracterizou a obstinação em evoluir o modelo burocrático. As propostas do NPM avançaram, pois os gastos e déficits públicos eram crescentes, em particular com as obras públicas. A reformulação da gestão pública segue com a proposta da inserção de práticas flexíveis, padronizando o ciclo de investimentos, da gestão privada no setor público (MOTTA, 2013).

De acordo com Luu et al. (2008), a gestão por resultado é o centro da melhoria incessante, que vem desenvolvendo no setor público procedimentos e métodos específicos para gerenciar projetos (GASIK, 2016).

Em vista do foco em resultados que a alta gestão persegue, deve ocorrer o conhecimento e controle das informações, convém capturar, armazenar, reutilizar e gerar conhecimento, formando o registro do conhecimento como uma fundação para aumentar sistematicamente o resultado positivo, sem desperdício de recursos e efeito prejudicial para 
a qualidade (KIRSCH, HINE E MAYBURY, 2015; WATTHANANON, MINGKHWAN, 2012; YUSOF, HASSAN E BAKAR, 2012).

No ambiente público a inserção ocorre com a reformulação o Ministério do Planejamento, Orçamento e Gestão (MPOG) onde divulgou um instrumento de autoavaliação dentre as dimensões de efetividade, eficácia e eficiência da gestão pública que em virtude do Programa Nacional de Gestão Pública e Desburocratização (GESPÚBLICA) foi instituído pelo Decreto 5.378/2005.

Para as autarquias a Lei $\mathrm{n}^{-}$8.666/93 condiciona a escolha da modalidade de licitação, que para obras e serviços de engenharia transita entre a concorrência, tomada de preços e carta convite e por último a dispensa de licitação, e ainda pela Lei $\mathrm{n}^{0}$ 12.462/11, que institui o Regime Diferenciado de Contratações Públicas (RDC).

Com isso, há obras de sucesso com seu perfeito desempenho técnico, mantendo-se a programação e os custos orçamentais, eficazmente e eficientemente gerenciados e há outras obras que incorre em atraso e saturações de custos, as quais se relacionam ao projeto, ao local, ao processo, às pessoas e às questões técnicas, legalmente autorizada ao limite da lei, porém, ocorre aditivo ao contrato, acrescendo o prazo e valor (DOLOI et al., 2012; FRIMPONG, OLUWOYE E CRAWFORD, 2003).

Kaliba, Muya e Mumba (2009); Philippsen Junior e Fabrício (2011), identificam que as variáveis de maior frequência entre as causas de erros ou falhas estão relacionadas as questões relativas à interação entre projetistas, projeto e obra e tem como necessidades de ter atenção, cuidado e critério na etapa de elaboração dos orçamentos. Segundo Magnussen e Olsson (2006), outro fator que causa aditivos são os atrasos, pois os preços dos produtos sobem. Por outro lado, há os itens que são esquecidos ou omitidos da planilha orçamentária e que causam aditivos nos contratos, fator que os autores atribuem à incompetência.

Perante os erros ou falhas o planejamento aos níveis: estratégico, tático e operacional; torna-se uma ferramenta gerencial que assegura racionalidade a tomada de decisões, direcionando esforços e ações, visando atingir os objetivos projetados, por meio de critérios técnicos, e moderação dos riscos no processo de execução. A gestão estratégica por meio do controle de suas ações e objetivos estruturados nos conhecimentos, e reorganizando e redesenhado a formação de processos para melhoria, e a fim contemplar a eficiência e eficácia, é considerado uma forma sustentável da aplicação de recursos públicos na criação de valores organizacionais (BRYSON, CROSBY E BRYSON, 2009; FOOLADVAND, YARMOHAMMADIAN E SHAHTALEBI, 2015; KAHVECI et al., 2012).

Assim, a gestão estratégica - strategic management - tornou-se a peça-chave evoluindo para o performance management como um processo de gerenciamento para resultados, reunindo a prática de mensuração de resultados com as práticas governamentais do setor público e do planejamento estratégico (PUTHAMONT, CHAROENNGAM, 2007; BEUREN, 2014). 
Behn (2003) e Soibelman et al. (2005), relatam que o benchmarking como técnica utilizada para comparar procedimentos e o desempenho com as práticas recomendadas de concorrentes, reconhecimento de padrões, estatística e visualização para a extração de conceitos, correlações, possibilita a instituição o entendimento de como atingir melhores desempenhos, a fixar metas realistas com base na visão conjunta do ambiente interno e externo; pode ser aplicando a uma variação de indicadores financeiros e não-financeiros, com objetivo de entende-los, adaptá-los e implementá-los, além de priorizar áreas de melhoria (COSTA et al., 2005; KAPLAN E NORTON, 2005).

Com as dimensões de tempo/cronograma, do orçamento, qualidade e satisfação pública que são critérios primordiais na avaliação de desempenho, e contribuições para o bem-estar geral de seus usuários finais, essas dimensões formam a cadeia de valor e do desempenho a qual constitui as dimensões de esforço e resultado, (AHMAD et al., 2016; BRASIL, 2009; COX, ISSA E AHRENS, 2003; TOOR, OGUNLANA, 2010; YUN et al., 2015). Logo, os indicadores pelo resultado vêm dos esforços em distintas dimensões, peculiar a organização e vinda de um construto específico do setor (Palvarini, 2010).

Segundo Beatham et al. (2004) e Ho (2011), os KPI podem reestruturar o planejamento das atividades operacionais e realocar os recursos, porém deve refletir as características do país, da empresa em situações de mercado diferentes, por possuir políticas e estratégias, culturas e ambientes competitivos, requisitando mensurações diferenciadas, e a solução de alguns dos problemas a serem solucionados no gerenciamento do setor de obras.

Por conseguinte, os KPI são as compilações de dados e medidas correlacionados e definidas por resultados quantitativos de um processo da construção civil, como \$/unidade, ou por medidas qualitativas, em que se verifica a satisfação do cliente. Portanto os principais indicadores passam a acessar o desempenho operacional da construção (COX, ISSA E AHRENS, 2003). Para medir o desempenho e a aplicação da abordagem de avaliação comparativa, primeiro devem ser estabelecidos os KPI, que são a chave na determinação do sucesso global da organização (ABD et al., 2013). Segundo Nasir et al. (2012), as métricas são um conjunto de ferramentas de medição para quantificar os resultados e podem ser realizadas concomitantemente ao ajustamento do KPI.

A sistematização dos principais modelos existentes de KPIs aplicados à Indústria da Construção (IC), pode-se destacar a síntese dos seis sistemas de Benchmarking, no Quadro 1, com os respectivos encarregados por sua elaboração. 
QUADRO 1 - Sistemas de Benchmarking

\begin{tabular}{|l|l|}
\hline Sistema de Benchmarking & Responsavél pelo Desenvolvimento / (País) \\
\hline $\begin{array}{l}\text { 1. KPI - Key Performance Indicators } \\
\begin{array}{l}\text { 2. SISIND-NET - Sistema de Medição de Desempenho } \\
\text { para benchmarking }\end{array}\end{array}$ & $\begin{array}{l}\text { Construction Excellence - UK Construction Best Practice } \\
\text { Programme/ (Reino Unido) } \\
\text { (NORIE) / (Brasil) }\end{array}$ \\
\hline 3. BEC-Centro de benchmarking do Setor da Construção & Byggeriets Evaluerings Center / (Dinamarca) \\
\hline $\begin{array}{l}\text { 4. ICBENCH - Indicadores de Desempenho e e } \\
\text { Produtividade - IDPs }\end{array}$ & $\begin{array}{l}\text { Faculdade de Engenharia da Universidade do Porto } \\
\text { / Portugal) }\end{array}$ \\
\hline $\begin{array}{l}\text { 5. NBS - Sistema Nacional de benchmarking } \\
\text { Corporación de Desarrollo Tecnológico e Programa de } \\
\text { Excelência em Gestão da Produção da Pontifícia } \\
\text { Universidade Católica do Chile (GEPUC) / (Chile) }\end{array}$ \\
\hline 6. CII BM\&M - Sistema Nacional de benchmarking & $\begin{array}{l}\text { Construction Industry Institute benchmarking and } \\
\text { Metrics / (Estados Unidos da América) }\end{array}$ \\
\hline
\end{tabular}

\section{FONTE: Adaptado de PINHEIRO (2011).}

A revisão da literatura mostra que a medida de desempenho da IC segue as medidas tradicionais como custo, tempo e qualidade, além de acrescer medidas qualitativas. Neste estudo é feita uma tentativa de capturar a métricas de diferentes KPIs, entre quantitativas e qualitativas.

Com a utilização da correlação de Pearson $(r)$ pode-se estimar e mensurar entre diferentes variáveis a direção e o grau da relação linear entre duas variáveis quantitativas, portanto o $r$ é uma medida de associação linear entre variáveis. As variáveis tidas como principais e operacionais, uma vez que são extraídas, ocorre a verificação do cruzamento das medidas, os atributos de cada variável, formando coletivamente uma explicação da mesma dentro das dimensões do objeto de estudo (DOLOI, 2009).

No entanto, uma regra se aplica a maioria das situações com as seguintes faixas: se $r<0,20$, a correlação é negligenciável; se 0,20 $\leq r<0,40$, a correlação é fraca; se o,40 $\leq(r)<0,60$, a correlação é moderada; se $0,60 \leq r<0,80$, a correlação é forte; se $r \geq 0,80$, a correlação é muito forte (FRANZBLAU, 1958).

A matriz de correlação linear de Pearson entre as variáveis, terá a significância representada pela probabilidade $(p)$ de rejeição da hipótese; portanto, serão consideradas as inferências em que os coeficientes de correlação de Pearson, entre os pares das matrizes sejam significativos e de alta magnitude, com $r \geq 0,6$ e $p \leq 0,05$, (FILHO E JUNIOR; 2009; VOELKL E GERBER, 1999). 
A escolha do modelo de análise por envoltória de dados (DEA) está inserido no contexto de avaliação da eficiência técnica e permite comparar o desempenho de unidades de produção - Decision Making Units (DMU) - pertencentes as diferentes instituições, sendo assim o DEA uma metodologia determinística e não paramétrica (RAMÍREZ et al., 2004).

A DEA usa todos os dados disponíveis para construir uma fronteira empírica de melhores práticas, cada DMU ineficiente é comparada, e permite que cada unidade identifique um grupo de benchmarking que seguem os mesmos objetivos e prioridades das DMU que se desempenham melhor (AMADO et al., 2011).

A esse respeito, a DEA visa respeitar as prioridades de cada DMU, permitindo que cada uma delas escolha a estrutura de pesos para insumos e produtos que mais beneficiam sua avaliação. Como resultado, pretende ranquear cada unidade da melhor forma possível em comparação com as outras unidades (MELLO et al., 2005).

Existem basicamente dois modelos clássicos de DEA: o modelo de escala de retorno constante - Constant Returns To Scale (CRS), também conhecido como Charnes, Cooper e Rhodes (CCR) (Charnes, Cooper e Rhodes, 1978) - e o modelo de escala de retorno variável Variable Returns To Scale (VRS), ou Banker, Charnes e Cooper (BCC) (BANKER, CHARNES E COOPER, 1984).

O modelo BCC/VRS é independente da escala de operação e, assim, passa a "envelopar" as unidades ineficientes para cada escala de produção, serve também como referência para o estabelecimento de metas eficientes (benchmarks) (BELLONI, 2000; CASADO, 2007; LINS et al., 2007).

A medida de eficiência técnica é comparada pela perspectiva do produto com diferentes sentidos, quais sejam: 1 . a eficiência técnica com retornos constantes de escala, vista como eficiência técnica produtiva; 2. a eficiência técnica pura que traz retornos variáveis de escala; e 3. a relação entre as duas anteriores, definida como eficiência de escala (CASA NOVA, 2002; PEÑA, 2008).

\section{PROCEDIMENTOS METODOLÓGICOS}

No Estado de Santa Catarina, envolvendo suas IFES autárquicas, dentre elas a Universidade Federal de Santa Catarina (UFSC), a Instituição Federal de Santa Catarina (IFSC) e Instituto Federal Catarinense (IFC), numa totalidade de 43 Campi com finalização da construção, reforma e ampliação, nos anos de 2015 e 2016.

O IFC com 9 obras que foram analisadas devido ao enquadramento dessas estarem relacionadas as problemáticas da pesquisa. Já a UFSC com 10 obras e, por fim, o IFSC com 19 obras, o que computa 38 obras na problemática da pesquisa.

São oito variáveis entre entradas e saídas que foram utilizadas para correlação de Pearson e análise por envoltória de dados. Todas as variáveis têm ligação com as problemáticas. Para as métricas, são 
formadas por dados que se limitam a projetos executados nos dois últimos anos de exercícios fiscais (EL-MASHALEH; EDWARD MINCHIN JR; O’BRIEN, 2007).

As entradas foram dadas pelas variáveis: Valor estimado para a obra (VE), Valor da contratação da obra (VC), Área construída da obra $\left(\mathrm{M}^{2}\right)$ e Tempo estimado para a obra (TE); já as saídas pelas variáveis: Tempo resultante da soma do TE mais VAT - (TR), Valor aditivado de acréscimo de serviço (VAS), Valor aditivado de tempo (VAT) e Valor resultante da soma de VC mais VAS - (VR).

\section{RESULTADOS E DISCUSSÕES}

Nesta pesquisa foi realizada uma avaliação para se decidir quais os KPIs mais relevantes da seleção indicada no referencial teórico. A validação dos KPIs propostos foi efetuada com a correlação de Pearson.

Assim, após os resultados da correlação de Pearson foi elaborado um quadro com as dimensões, subdimensões e métricas firmadas como KPIs do IFC. Já após a análise por envoltória de dados foram definidos as metas e o benchmarking a ser utilizado pelo IFC.

Inicialmente, o IFC foi analisado pelos seus dados que constam a correlação entre as variáveis com os valores variando entre 0,183 a 0,999. A fraca correlação apresentada demonstra que o desempenho em determinado resultado não influencia diretamente o outro, e não poderia ser incluído nas métricas com as demais variáveis. Enfim, o IFC foi analisado pelos dados que constam no Quadro 2 e as correlações aceitas foram as com grau de forte e muito forte correlação dentro de uma significância razoável, são as do Quadro 3:

QUADRO 2 - DMUs e variáveis do IFC

\begin{tabular}{|l|l|l|l|l|l|l|l|l|}
\hline DMU & $\mathrm{VE}^{1}$ & $\mathrm{VC}^{1}$ & $\mathrm{M}^{\prime \prime 1}$ & $\mathrm{TE}^{1}$ & $\mathrm{TR}^{1}$ & $\mathrm{VR}^{1}$ & $\mathrm{VAS}^{1}$ & VAT $^{1}$ \\
\hline IFC 1 & $3.961 .790,11$ & $3.920 .000,00$ & $2.687,83$ & 570 & 630 & $4.176 .921,66$ & $256.921,66$ & 60,00 \\
\hline IFC 2 & $59.551,05$ & $59.551,05$ & 391,64 & 105 & 105 & $59.551,05$ & 0,00 & 0,00 \\
\hline IFC 3 & $1.499 .503,70$ & $1.190 .000,01$ & 771,74 & 150 & 150 & $1.190 .000,01$ & 0,00 & 0,00 \\
\hline IFC 4 & $1.831 .686,95$ & $1.589 .714,57$ & $1.950,00$ & 364 & 800 & $1.960 .240,38$ & $370.525,81$ & 436,00 \\
\hline IFC 5 & $1.000 .000,00$ & $1.475 .770,63$ & $1.181,26$ & 377 & 507 & $1.683 .848,12$ & $208.077,49$ & 130,00 \\
\hline IFC 6 & $648.691,80$ & $518.561,22$ & 298,10 & 225 & 367 & $518.561,22$ & 0,00 & 142,00 \\
\hline IFC 7 & $9.059 .866,25$ & $7.466 .319,58$ & $5.577,39$ & 547 & 744 & $8.427 .810,84$ & $961.491,26$ & 197,00 \\
\hline IFC 8 & $2.128 .379,51$ & $2.118 .546,12$ & $1.787,67$ & 288 & 483 & $2.243 .463,04$ & $124.916,92$ & 195,00 \\
\hline IFC 9 & $3.227 .074,14$ & $2.599 .197,87$ & $2.546,18$ & 540 & 785 & $2.705 .709,43$ & $106.511,56$ & 245,00 \\
\hline
\end{tabular}

FONTE: Elaborado pelo autor (2019). 


\section{Revista de Gestão Pública}

PRÁTICAS E DESAFIOS ISSN: 2177-1243

Notas:

As variáveis do quadro são descritas a seguir:

VE - Valor estimado para a obra

VC - Valor da contratação da obra

$\mathrm{M}^{2}$ - Área construída da obra

TE - Tempo estimado para a obra

TR - Tempo resultante da soma do TE mais VAT

VAS - Valor aditivado de acréscimo de serviço

VAT - Valor aditivado de tempo

VR - Valor resultante da soma de VC mais VAS

QUADRO $_{3}$ - Correlações aceitas do IFC

\begin{tabular}{|c|c|c|c|c|c|c|}
\hline & VE & $\mathrm{VC}$ & $\mathrm{M}^{2}$ & TE & TR & VR \\
\hline VC & $0,991^{* *}$ & 1 & & & & \\
\hline $\mathrm{M}^{2}$ & $0,980^{* *}$ & $0,979^{* *}$ & 1 & & & \\
\hline $\mathrm{TE}$ & $0,727^{*}$ & $0,769^{*}$ & $0,794^{*}$ & 1 & & \\
\hline TR & & & $0,706^{*}$ & $0,878^{* *}$ & 1 & \\
\hline VR & $0,991^{* *}$ & $0,999^{* *}$ & $0,982^{* *}$ & $0,760^{*}$ & & 1 \\
\hline VAS & $0,901^{* *}$ & $0,901^{* *}$ & $0,919^{* *}$ & & & $0,922^{* *}$ \\
\hline VAT & & & & & $0,789^{*}$ & \\
\hline \multicolumn{7}{|c|}{ **. A correlação é significativa no nível 0,01 (2 extremidades). } \\
\hline *. A c & sioni & no níve & extrer & & & \\
\hline
\end{tabular}

FONTE: Elaborado pelo autor (2019).

Notas:

As variáveis do quadro são descritas a seguir:

VE - Valor estimado para a obra

VC - Valor da contratação da obra

$\mathrm{M}^{2}$ - Área construída da obra

TE - Tempo estimado para a obra

TR - Tempo resultante da soma do TE mais VAT

VAS - Valor aditivado de acréscimo de serviço 
Revista de Gestão Pública

VAT - Valor aditivado de tempo

VR - Valor resultante da soma de VC mais VAS

Por conseguinte, a UFSC foi analisada por meio dos seus dados que evidencia uma correlação entre as variáveis com os valores variando entre 0,053 a 0,999. A UFSC foi analisada pelos dados que constam no Quadro 4 e as correlações aceitas com o grau de forte e muito forte correlação dentro de uma significância razoável estão discriminadas no Quadro 5.

QUADRo 4 - DMUs e variáveis da UFSC

\begin{tabular}{|l|l|l|l|l|l|l|l|l|}
\hline DMU & VE & VC & $\mathrm{M}^{2}$ & TE & TR & VR & VAS & VAT \\
\hline UFSC 1 & $4.279 .599,85$ & $4.279 .599,85$ & $2.755,53$ & 240 & 465 & $5.295 .894,49$ & $1.016 .294,64$ & 225,00 \\
\hline UFSC 2 & $675.700,87$ & $564.210,13$ & 593,20 & 120 & 300 & $685.632,04$ & $121.421,91$ & 180,00 \\
\hline UFSC 3 & $139.339,59$ & $136.666,63$ & 307,86 & 270 & 345 & $197.946,01$ & $61.279,38$ & 75,00 \\
\hline UFSC 4 & $686.266,20$ & $597.737,86$ & 213,84 & 360 & 391 & $597.737,86$ & 0,00 & 31,00 \\
\hline UFSC 5 & $1.383 .040,56$ & $1.193 .564,00$ & $7.716,14$ & 360 & 420 & $1.296 .836,95$ & $103.272,95$ & 60,00 \\
\hline UFSC 6 & $13.403 .724,69$ & $11.206 .685,93$ & $4.611,69$ & 974 & 1.064 & $13.095 .521,80$ & $1.888 .835,87$ & 90,00 \\
\hline UFSC 7 & $3.362 .198,22$ & $2.689 .758,58$ & $1.273,28$ & 420 & 479 & $2.738 .134,14$ & $48.375,56$ & 59,00 \\
\hline UFSC 8 & $2.652 .853,26$ & $2.268 .189,54$ & 772,67 & 360 & 480 & $2.392 .852,04$ & $124.662,50$ & 120,00 \\
\hline UFSC 9 & $27.413,62$ & $22.399,67$ & 358,17 & 29 & 29 & $22.399,67$ & 0,00 & 0,00 \\
\hline $\begin{array}{l}\text { UFSC } \\
\text { 10 }\end{array}$ & $137.525,45$ & $122.397,65$ & 42,89 & 89 & 109 & $127.920,85$ & $5.523,20$ & 20,00 \\
\hline
\end{tabular}

Notas:

As variáveis do quadro são descritas a seguir:

VE - Valor estimado para a obra

VC - Valor da contratação da obra

$\mathrm{M}^{2}$ - Área construída da obra

TE - Tempo estimado para a obra

TR - Tempo resultante da soma do TE mais VAT

VAS - Valor aditivado de acréscimo de serviço

VAT - Valor aditivado de tempo

VR - Valor resultante da soma de VC mais VAS 


\section{Revista de Gestão Pública}

QUADRO 5 - Correlações aceitas da UFSC

\begin{tabular}{|c|c|c|c|c|c|}
\hline & VE & $\mathrm{VC}$ & TE & TR & VR \\
\hline VE & 1 & & & & \\
\hline VC & $0,998^{* *}$ & 1 & & & \\
\hline TE & $0,903^{* *}$ & $0,887^{* *}$ & 1 & & \\
\hline TR & $0,923^{* *}$ & $0,918^{* *}$ & $0,967^{* *}$ & 1 & \\
\hline VR & $0,995^{* *}$ & $0,999^{* *}$ & $0,872^{* *}$ & $0,909^{* *}$ & 1 \\
\hline VAS & $0,939^{* *}$ & $0,955^{* *}$ & $0,761^{*}$ & $0,828^{* *}$ & $0,968^{* *}$ \\
\hline \multicolumn{6}{|c|}{ **. A correlação é significativa no nível 0,01 (2 extremidades). } \\
\hline \multicolumn{6}{|c|}{ *. A correlação é significativa no nível 0,05 (2 extremidades). } \\
\hline
\end{tabular}

FONTE: Elaborado pelo autor (2019).

Notas:

As variáveis do quadro são descritas a seguir:

VE - Valor estimado para a obra

VC - Valor da contratação da obra

$\mathrm{M}^{2}$ - Área construída da obra

TE - Tempo estimado para a obra

TR - Tempo resultante da soma do TE mais VAT

VAS - Valor aditivado de acréscimo de serviço

VAT - Valor aditivado de tempo

VR - Valor resultante da soma de VC mais VAS

Como se pode ver, o Quadro 4 evidencia uma correlação entre as variáveis com os valores variando entre -0,056 a 1,o0, de fraca a muito fortemente correlacionada, o que as tornam direta e indiretamente proporcionais, nas relações em que $\mathrm{r}$ ficou próxima a zero, portanto, praticamente nula, formou uma correlação negligenciável. Por fim, o IFSC foi analisado pelos dados que constam no Quadro 6 e as correlações aceitas com o grau de forte e muito forte correlação dentro de uma significância razoável são as do Quadro 7:

QUADRo 6 - DMUs e variáveis do IFSC 
Revista de Gestão Pública

\begin{tabular}{|l|l|l|l|l|l|l|l|l|}
\hline DMU & VE & VC & $\mathrm{M}^{2}$ & TE & TR & VR & VAS & VAT \\
\hline IFSC 1 & $7.950 .000,00$ & $7.950 .000,00$ & $6.500,00$ & 426 & 471 & $7.950 .000,00$ & 0,00 & 45,00 \\
\hline IFSC 2 & $2.055 .000,00$ & $2.055 .000,00$ & 750,00 & 360 & 390 & $2.055 .000,00$ & 0,00 & 30,00 \\
\hline IFSC 3 & $1.798 .454,53$ & $1.798 .454,53$ & 500,00 & 390 & 450 & $1.798 .454,53$ & 0,00 & 60,00 \\
\hline IFSC 4 & $1.720 .287,73$ & $1.720 .287,73$ & $1.315,00$ & 390 & 416 & $1.720 .287,73$ & 0,00 & 26,00 \\
\hline IFSC 5 & $542.457,82$ & $542.457,82$ & 286,00 & 150 & 210 & $542.457,82$ & 0,00 & 60,00 \\
\hline IFSC 6 & $766.591,40$ & $766.591,40$ & $1.811,29$ & 47.369 & 47.459 & $766.591,40$ & 0,00 & 90,00 \\
\hline IFSC 7 & $2.013 .489,69$ & $2.013 .489,69$ & $1.315,00$ & 390 & 390 & $2.013 .489,69$ & 0,00 & 0,00 \\
\hline IFSC 8 & $234.733,69$ & $231.330,06$ & 270,00 & 180 & 180 & $231.330,06$ & 0,00 & 0,00 \\
\hline IFSC 9 & $4.026 .561,72$ & $3.479 .077,67$ & $2.073,35$ & 450 & 450 & $3.479 .077,67$ & 0,00 & 0,00 \\
\hline IFSC 10 & $1.012 .183,56$ & $991.939,88$ & 650,00 & 240 & 300 & $991.939,88$ & 0,00 & 60,00 \\
\hline IFSC 11 & $62.659,36$ & $54.388,32$ & 35,00 & 180 & 180 & $54.388,32$ & 0,00 & 0,00 \\
\hline IFSC 12 & $59.086,95$ & $50.164,82$ & 35,00 & 180 & 180 & $50.164,82$ & 0,00 & 0,00 \\
\hline IFSC 13 & $293.392,07$ & $261.705,72$ & 600,00 & 180 & 180 & $261.705,72$ & 0,00 & 0,00 \\
\hline IFSC 14 & $102.000,00$ & $91.121,40$ & 200,00 & 240 & 240 & $91.121,40$ & 0,00 & 0,00 \\
\hline IFSC 15 & $815.475,68$ & $754.315,01$ & 465,00 & 240 & 270 & $754.315,01$ & 0,00 & 30,00 \\
\hline IFSC 16 & $246.312,61$ & $221.558,19$ & 245,68 & 240 & 240 & $221.558,19$ & 0,00 & 0,00 \\
\hline IFSC 17 & $334.987,34$ & $288.089,12$ & 232,00 & 135 & 135 & $288.089,12$ & 0,00 & 0,00 \\
\hline IFSC 18 & $93.830,31$ & $90.358,59$ & $2.000,00$ & 135 & 135 & $90.358,59$ & 0,00 & 0,00 \\
\hline IFSC 19 & $57.016,80$ & $56.959,78$ & 80,00 & 150 & 150 & $56.959,78$ & 0,00 & 0,00 \\
\hline
\end{tabular}

FONTE: Elaborado pelo autor (2019)

Notas:

As variáveis do quadro são descritas a seguir:

VE - Valor estimado para a obra

VC - Valor da contratação da obra

$\mathrm{M}^{2}$ - Área construída da obra

TE - Tempo estimado para a obra

TR - Tempo resultante da soma do TE mais VAT

VAS - Valor aditivado de acréscimo de serviço

VAT - Valor aditivado de tempo 


\section{Revista de Gestão Pública}

VR - Valor resultante da soma de VC mais VAS

QUADRO 7 - Correlações aceitas do IFSC

\begin{tabular}{|c|c|c|c|c|}
\hline & VE & VC & M2 & TE \\
\hline VE & 1 & & & \\
\hline $\mathrm{VC}$ & $0,998^{* *}$ & 1 & & \\
\hline M2 & $0,897^{* *}$ & $0,905^{* *}$ & 1 & \\
\hline TR & & & & $1,000^{* *}$ \\
\hline VR & $0,998^{* *}$ & $1,000^{* *}$ & $0,905^{* *}$ & \\
\hline \multicolumn{5}{|c|}{ **. A correlação é significativa no nível 0,01 (2 extremidades). } \\
\hline \multicolumn{5}{|c|}{ *. A correlação é significativa no nível 0,05 (2 extremidades). } \\
\hline
\end{tabular}

Notas:

As variáveis do quadro são descritas a seguir:

VE - Valor estimado para a obra

VC - Valor da contratação da obra

$\mathrm{M}^{2}$ - Área construída da obra

TE - Tempo estimado para a obra

TR - Tempo resultante da soma do TE mais VAT

VAS - Valor aditivado de acréscimo de serviço

VAT - Valor aditivado de tempo

VR - Valor resultante da soma de VC mais VAS

De acordo com Brasil (2009) e Souza (2018), que entendem que os indicadores são divididos entre eficiência (produtividade, tempo e custo): medem a proporção de recursos consumidos com relação às saídas dos processos; eficácia (qualidade e quantidade): focam as características do produto pelo serviço realizado; efetividade (impacto interno e externo): focam as consequências do produto pelo serviço. Nesta pesquisa o indicador efetividade tem o objetivo nas mudanças quantitativas, para pesquisas futura o subjetivo (estado de espírito) ou substantivo (qualitativo). Assim, foram elaborados indicadores específicos dentre as dimensões de efetividade, eficácia e eficiência e suas consecutivas subdimensões, extraídos dos sistemas de benchmarking e compilados a GESPÚBLICA. A Tabela I demonstra o relacionamento das variáveis no formato de métricas: 


\section{Revista de Gestão Pública}

PRÁTICAS E DESAFIOS

ISSN: 2177-1243

TABELA 1 - Proposta dos KPIs

\begin{tabular}{l}
\hline DIMENSÕES DE \\
DESEMPENHO \\
(INDICADORES)
\end{tabular}

\section{(INDICADORES)}

EFETIVIDADE

Por meio da resposta a um questionário,

\section{EFETIVIDADE}

\section{IMPACTO EXTERNO}

utilizando uma escala de 1 (= totalmente

insatisfeito) a 10 (= totalmente satisfeito).

\begin{tabular}{ll}
\hline \multirow{2}{*}{ EFICÁCIA } & QUANTIDADE \\
\cline { 2 - 2 } & QUALIDADE $(Q)$ \\
\hline \multirow{2}{*}{ EFICIÊNCIA } & PRODUTIVIDADE \\
\cline { 2 - 2 } & TEMPO \\
\hline FONTE: Elaborada pelo autor (2019).
\end{tabular}

FONTE: Elaborada pelo autor (2019).

Notas:

As variáveis da tabela são descritas a seguir:

VE - Valor estimado para a obra

VC - Valor da contratação da obra

$\mathrm{M}^{2}$ - Área construída da obra

TE - Tempo estimado para a obra

TR - Tempo resultante da soma do TE mais VAT

VAS - Valor aditivado de acréscimo de serviço

VAT - Valor aditivado de tempo

VR - Valor resultante da soma de VC mais VAS

A Tabela 2 apresenta os resultados das dimensões de efetividade, eficácia e eficiência com uso dos dados da DMU mais eficiente, DMU IFSC 1. A subdimensão 'impacto externo' é a única a não produzir resultados, pois o questionário seria resoluto a DMU IFSC 1 em suas especificidades. 
TABELA 2 - Metas ao IFC

\begin{tabular}{llll} 
TABELA 2 & - Metas ao IFC & & \\
\hline \multirow{2}{*}{ DMU } & $\begin{array}{l}\text { DIMENSÕES DE } \\
\text { DESEMPENHO } \\
\text { (INDICADORES) }\end{array}$ & SUBDIMENSÕES & MÉTRICAS \\
\hline \multirow{2}{*}{ EFETIVIDADE } & & IMPACTO EXTERNO & QUESTIONÁRIO A SER REALIZADO \\
\cline { 2 - 4 } & EFICÁCIA 1 & IMPACTO INTERNO & R $\$ 1.223,08$ \\
\cline { 2 - 4 } & \multirow{2}{*}{ EFICIÊNCIA } & QUANTIDADE & $100,00 \%$ \\
\hline
\end{tabular}

\section{FONTE: Elaborada pelo autor (2019).}

Destaca-se para a dimensão da efetividade que seja realizado um questionário específico para a obra realizada. O questionário deve ser proposto, a fim de captar informações da parte interessada externa, focado na sociedade, possibilitando observar o grau de satisfação com o patrimônio público, avaliando então o desempenho no 'impacto externo'.

Foi verificado que VR e M² são relacionáveis, pois o valor resultante da obra depende da área a ser construída. Resultantes da análise de correlação realizada no IFSC, apresentaram uma correlação muito forte, permitindo a orientação da métrica $\left(\mathrm{VR} / \mathrm{M}^{2}\right)$ da subdimensão 'impacto interno'. Os resultados consideraram importante e como meta a presença de um valor igual ou abaixo de R\$1.223,08, menor que o CUB/SC referentes aos anos fiscais de 2015 e 2016, na DMU IFSC 1, o que indica uma economia na realização da obra. $\mathrm{O}$ resultado apresentado incentiva a construção e o aprimoramento de inventários de recursos, e acolhe o ideal da redução na utilização das entradas para obtenção dos melhores resultados em suas saídas.

Já o VC e VR são associáveis, pelo motivo do valor resultante depender do valor que a obra foi contratada, utilizou-se dessas variáveis após a análise do IFSC, da UFSC e do IFC, e são aceitas pelo grau de muito fortemente correlacionadas, no qual incorreu na métrica: (VC/VR) *100, da dimensão da eficácia, em se tratando da subdimensão de 'quantidade', houve resultado de $100,00 \%$. Isto representa que o trabalho foi realizado exatamente igual ao que havia sido contratado. Portanto, o valor da obra deve ser igual ao do contratado, portanto, a meta fica definida pela porcentagem de 100,00\%.

Por conseguinte, VAS e VR são conectáveis pelo fato do valor de aditivo de acréscimo de serviço impactar diretamente no valor resultante da obra. Essas variáveis são utilizadas para verificar a subdimensão 'qualidade', obtidos da análise 
da UFSC e do IFC, apresentou uma interpretação de correlação de grau muito forte, o que incidiu numa métrica: (VAS/VR) * 100 . Há perda de recursos quando disposto o aditivo de acréscimo de serviço, portanto resultando num valor maior de contrato. O que requer de forma corretiva uma meta de $0,00 \%$ de aditivo.

Consequentemente, VE e VR são concatenados devido ao valor estimado da obra ser a entrada referencial da licitação e guia o valor resultante da obra na saída. Empregados para verificar a subdimensão 'produtividade', obtidos na análise do IFSC, da UFSC e do IFC, com a interpretação de uma correlação de grau muito forte, sucedeu a métrica: (VE/VR) *100, importando que a meta da 'produtividade' seja de $100 \%$, entendo assim que não venha a ocorrer paralisação ou redução do objeto licitado.

À vista disso, a subdimensão 'custo' transcorreu na métrica: [(VR-VE) /VE] ${ }^{*} 100$, com meta de $0 \%$ por possibilitar a redução perdas com os aditivos de acréscimo, perante o prazo dilatado, o que significa que o trabalho realizado tem o valor estimado exatamente igual ao valor resultante da obra. Por fim, o 'custo' ficou com a interação entre valor resultante e o valor estimado da obra, dando ao planejamento orçamentário e à produção de projetos a máxima eficiência quando apresentados resultados idênticos nessas variáveis de entrada e de saída.

Contudo, TE e VAT são correlatáveis porque o tempo estimado da obra serve de entrada referencial e o valor aditivado de acréscimo de tempo é uma saída prorrogadora a variável de entrada. Obtidos na análise do IFC, assumem a interpretação de uma correlação de grau forte. Já o TE e TR são confrontáveis, uma vez que o tempo estimado de obra é uma entrada referencial para saída: tempo resultante de obra. Encontrados na análise da UFSC e do IFC, interpretados numa correlação de grau muito forte, e na análise do IFSC apresentaram uma correlação positivamente perfeita, as variáveis intercorreram na métrica da subdimensão 'tempo': [(TR - TE/ TE)) * ${ }^{*}$ 10o].

A 'produtividade' da subdimensão na dimensão eficiência, a DMU foi $100,00 \%$, indicando que se produziu exatamente o que foi previsto, onde a entrada dada pelo valor estimado da obra minimamente coincida com o produto - valor resultante da obra, e que possibilite a manutenibilidade do desconto dado pela contratada no ato da licitação, gerando maior produtividade, consequentemente maior eficiência.

Porém, no caso da subdimensão 'tempo', houve uma dilatação para que fosse cumprida a execução do objeto licitado: acréscimo de 10,56\% no tempo estimado para a obra, fator esse complexo de planejar com a diversidade do itinerário da construção civil nacional, permite a possibilidade de se estende-lo haja vista a interação entre tempo resultante e tempo estimado. Faz-se necessário, assim, identificar a fonte de perda e prevenir futuras perdas de prazo, assim como corrigir o ritmo inadequado.

No desenvolvimento do método do envelope não orientado, e priorizando as entradas, as DMUs eficientes, com escore igual a 1, são 
do resultado pelo método BCC dual. Toda a sequência de benchmark $(\lambda)$ recomenda em taxas proporcionais as DMUs que tiveram maior importância na análise da DMU ineficiente.

A análise da comparação no escore de eficiência técnica, com escore igual a 1, para identificar os possíveis efeitos sobre as dimensões mensuradas, e ajustar a variação de escala, observando o Quadro 5:

QUADRO 5 - Eficiência técnica
\begin{tabular}{|l|l|l|l|l|}
\hline DMU & $\begin{array}{l}\text { Escore de eficiência } \\
\text { técnica }\end{array}$ & $\begin{array}{l}\text { Escore de eficiência } \\
\text { técnica pura }\end{array}$ & $\begin{array}{l}\text { Escore de eficiência de } \\
\text { escala }\end{array}$ & $\begin{array}{l}\text { Retornos } \\
\text { de escala }\end{array}$ \\
\hline UFSC 3 & 1 & 1 & 1 & Constante \\
\hline IFC 4 & 1 & 1 & 1 & Constante \\
\hline IFC 5 & 1 & 1 & 1 & Constante \\
\hline IFC 6 & 1 & 1 & 1 & Constante \\
\hline IFSC 3 & 1 & 1 & 1 & Constante \\
\hline UFSC 1 & 1 & 1 & 1 & Constante \\
\hline UFSC 2 & 1 & 1 & 1 & Constante \\
\hline UFSC 10 & 1 & 1 & 1 & Constante \\
\hline UFSC 6 & 1 & 1 & 1 & Constante \\
\hline UFSC 8 & 1 & 1 & 1 & Constante \\
\hline IFSC 6 & 1 & 1 & 1 & Constante \\
\hline
\end{tabular}

FONTE: Elaborado pelo autor (2019).

Nas DMUs que conseguiram ser eficientes em todas as técnicas, o retorno de escala é constante. Demonstra que a escala de produção não influenciou, haja visto que as IFES são instituições sem fins lucrativos. Não é dada ênfase ao quanto é produzido e sim que se obtenham os melhores índices de eficácia, eficiência e efetividade.

O benchmarking auxiliou a direcionar as IFES a utilização da modalidade de licitação (RDC). Identifica-se que a modalidade de licitação alinhada ao contrato do objeto licitado leva as empresas terceirizadas a produzirem de forma eficiente, colaborando com a gestão por resultados. Portanto, as entradas, variáveis planejadas, devem permanecer iguais ou próximas às saídas, variáveis estas resultantes do produto executado. Isso contribui para que o RDC seja a melhor modalidade de licitação a ser escolhida no processo licitatório.

Assim, o planejamento estratégico institucional e o setorial, de que deriva a realização do planejamento tático e operacional, bem como sua estrutura organizacional e de planejamento, deve ser levantado pelo inventário de recursos. Estes precisam ser alinhados à missão, à visão e aos objetivos estratégicos, assim como possibilitar uma maior eficiência de escala, permitindo que a gestão estratégica obtenha êxito em seus resultados. 
A DMU IFSC 1 não apresentou no comparativo das eficiências técnicas escore igual a $\mathbf{I}(\mathrm{um})$ para todas as técnicas, com exceção para eficiência de técnica pura. No entanto, representa retornos variáveis de escala e depura os efeitos da ineficiência decorrente do inadequado porte da unidade com a escala decrescente, indicando que se operou com altos valores das variáveis de entrada. Essa DMU é a primeira colocada no ranque da fronteira da eficiência pelos modelos do envelope e multiplicadores, além de ter menor índice de aditivos. Seus resultados são utilizados na métrica desenvolvida nesta pesquisa para serem utilizados como metas dirigidas.

Os resultados tornam-se uma estrutura básica de conhecimento, e fica explícita a necessidade de que o recurso organizacional e o gerenciamento, juntamente com os servidores, trabalhem em conjunto para que se possa atingir as metas.

\section{CONSIDERAÇÕES FINAIS}

A pesquisa conseguiu elaborar indicadores de desempenho como técnica gerencial para o SOP das autarquias, especificamente IFES. O trabalho recorreu à teoria da gestão por resultados, e consagrou-a como técnica gerencial, assegurado pelo KPI, como solucionadora da problemática identificada nesta pesquisa.

O monitoramento do desempenho de obras públicas em IFES é, comumente, realizado com foco apenas em custo e prazo, ignorando outros indicadores importantes para tomada de decisão que podem, direta ou indiretamente, afetar o resultado da gestão pública. As categorias das dimensões e subdimensões encontradas elevam o potencial de ganho de recursos pela gestão, assim como em qualidade no seu uso em face a sociedade.

Perante a apresentação dos resultados e suas discussões, verificou-se que a pesquisa conseguiu entender que os critérios de eficiência, eficácia e efetividade agregados aos indicadores da IC conseguem mensurar a gestão de obras públicas. Além de possibilitar à gestão de avaliar a sua atuação conforme a elaboração de KPI, além de seguir metas por meio do sistema de benchmarking. Portanto, a instituição é conduzida a alcançar resultados que elimina e/ou mitiga as problemáticas, assim como agir sobre a luz das melhores práticas o que leva ao sucesso da gestão.

Logo, a avaliação atingiu ao nível tático da instituição, e os indicadores não se tornam itens de controle operacional. No entanto, subsidia a gerência na observação e determinação das possíveis falhas. Os resultados auxiliam a alta gestão em buscar novas estratégias que venham a assumir os riscos na gestão de seus recursos.

No conjunto das IFES analisadas pela correlação de Pearson e DEA, foi possível apresentar as variáveis de entradas (VE, VC, $\mathrm{M}^{2}$ e TE) e saídas (TR, VR, VAS e VAT), como um sistema de entradas e saídas que transforma recursos (entradas) em resultados (saídas). O DEA, com fins de mensuração da eficiência comparativa no âmbito das IFES, rela- 
cionou-se como benchmarking a modalidade de licitação favorável para os processos licitatórios, o RDC.

Definiu-se a efetividade como a capacidade de se promover resultados pretendidos; a eficiência se denotou como competência para se produzir resultados com dispêndio mínimo de recursos e esforços; e a eficácia, por sua vez, remeteu-se às condições controladas e a resultados desejados das obras.

Destaca-se para a dimensão da efetividade que seja realizado um questionário específico para a obra realizada. O questionário deve ser proposto à parte interessada, a fim de se observar o grau de satisfação com a patrimônio público, avaliando então o desempenho no 'impacto externo'.

Já quanto ao 'impacto interno', relacionou-se o valor resultante da obra com a área a ser construída. Os resultados, portanto, considerou-se importante e como meta para os IFES a presença de um valor igual ou abaixo do CUB/SC para o ano fiscal levantado.

Quanto à eficácia, indicou-se que a 'quantidade', no campo do ideal, seja o valor resultante da obra igual ao valor contratado para a obra. Logo, a 'qualidade' traduz a perda de recursos quando há aditivo de acréscimo de serviço, portanto resultando num valor maior de contrato.

Por último, a eficiência, onde a entrada dada pelo valor estimado da obra minimamente coincida com o produto - valor resultante da obra, e que possibilite a manutenibilidade do desconto dado pela contratada no ato da licitação, gerando maior produtividade, consequentemente maior eficiência. Porém, para a subdimensão 'tempo', fator complexo de planejar com a diversidade do itinerário da construção civil nacional, permite a possibilidade de se estender, haja vista a interação entre tempo resultante e tempo estimado. Por fim, o 'custo' ficou com a interação entre valor resultante e o valor estimado da obra, dando ao planejamento orçamentário e à produção de projetos a máxima eficiência quando apresentados resultados idênticos nessas variáveis de entrada e de saída.

Propusemos um conjunto de indicadores com métricas e metas pré-definidas, que possibilita à instituição avaliar sistemicamente o desempenho da gestão do SOP. Portanto, a alta gestão e responsáveis pela estratégica cabe a esses mobilizar os recursos iniciais com que conta a instituição, e partem ao auxílio do planejamento tático, portanto, a gestão do setor direciona a escolha da modalidade de licitação e a regência dos contratos, regularizando as ações operacionais.

O ponto referencia, desse modo, competências e aprendizados gerados e herdados dos processos decisórios formais da instituição. Do ponto de vista prático, a instituição tem a necessidade de dispor de inventários completos dos recursos e das exigências de investimentos para manter ativo os recursos acumulados.

O possível registro do desempenho, por meio dos KPI construídos, em fase de implementação ou não, compreende-se como reserva de 
conhecimento útil que se pretende mobilizar. É possível, então, sintetizar essas informações referindo-se a elas como estratégias pretendidas.

\section{REFERÊNCIAS}

$\mathrm{ABD}, \mathrm{H}$. et al. Indicators for measuring performance of building construction companies in Kingdom of Saudi Arabia. Journal of King Saud University, Engineering Sciences, v. 25, n. 2, p. 125-134, 2013.

AHMAD, S. B. S. et al. A Review of Performance Measurement for Successful Concurrent Construction. Procedia - Social and Behavioral Sciences, v. 226, n. 1877, p. 447-454, 2016.

AMADO, C. A. F. et al. Integrating the Data Envelopment Analysis and the Balanced Scorecard approaches for enhanced performance assessment. Omega, v. 40, p. 390-403, 2011.

BANKER, R.D.; CHARNES, A.; COOPER, W.W. Some models for estimating technical scale inefficiencies in data envelopment analysis. Management Science, v. 30, n. 9, p. 1078-1092, 1984.

BEATHAM, S.; ANUMBA, C.; THORPE, T.; HEDGES, I. KPIs: a critical appraisal of their use in construction. Benchmarking: An International Journal, v. 11, n. 1, p. 93-117, 2004.

BEHN, R. D. Why Measure Performance? Different Purposes Require Different Measures. Public Administration Review, v. 63, n. 5, p. 586-606, 2003.

BELLONI, I. Uma Metodologia de Avaliação da Eficiência Produtiva de Universidades Federais Brasileiras. Tese de Doutorado, UFSC, 2000.

BEUREN, Ilse Maria; TEIXEIRA, Silvio Aparecido. Avaliação dos sistemas de controle gerencial em instituição de ensino superior com o performance management and control. Revista de Gestão da Tecnologia e Sistemas de Informação, v. 11, n. 1, p. 169-192, 2014.

BRASIL. MINISTÉRIO DO PLANEJAMENTO. Melhoria da gestão pública por meio da definição de um guia referencial para medição do desempenho da gestão, e controle para o gerenciamento dos indicadores de eficiência, eficácia e de resultados do programa nacional de gestão pública e desburocratiza ministério do planejamento: produto 1: mapeamento bibliográfico e do estado da arte sobre indicadores de gestão. Brasília: MP, 2009.

BYGGERIETS EVALUERINGS CENTER. (2002).

Site Institucional. Disponível em: <http:// www. 
byggeevaluering.dk>. Acesso em: 13 set. 2017.

BRYSON, J. M.; CROSBY, B. C.; BRYSON, J. K.

Understanding strategic planning and the formulation and implementation of strategic plans as a way of knowing: the contributions of Actor-Network Theory. International Public Management Journal, v. 12, n. 2, p. 172-207, 2009.

CASA NOVA, S.P.C. Utilização da análise por envoltória de dados (DEA) na análise de demonstrações contábeis. Tese de doutorado da Universidade de São Paulo, São Paulo, 2002.

CASADO, F. L. Análise envoltória de dados: conceitos, metodologia e estudo da arte na educação superior. Revista Sociais e Humanas, v. 20, n. 1, p. 59-71, 2007. CHARNES, A.; COOPER, W.W.; RHODES, E. Measuring the efficiency of decision-making units. European Journal of Operational Research, v. 2, p. 429-444, 1978.

CONSTRUCTION INDUSTRY INSTITUTE (CII). CII Benchmarking and Metrics Data Rep. 2000, CII, Tex. 2000.

CORPORACIÓN DE DESARROLLO TECNOLÓGICO (CDT). Sistema Nacional de Benchmarking na Indústria da

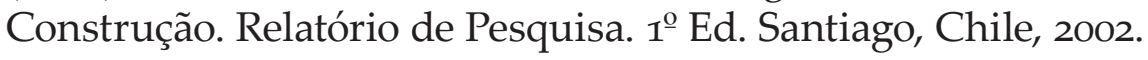

COSTA, D. B. et al. Sistema de indicadores para Benchmarking na construção civil. Porto Alegre: UFRGS/PPGEC/NORIE, 2005.

COX, R.F., ISSA, R.R.A., AHRENS, D. Management's perception of key performance indicators for construction. J. Constr. Eng. Manage. 129 (2), 142-151, 2003.

DOLOI, H. Analysis of pre-qualification criteria in contractor selection and their impacts on project success. Construction Management and Economics, 27, 1245-1263, 2009.

DOLOI, H.; et al. Analysing factors affecting delays in Indian construction projects. International Journal of Project Management. V. 30 p. 47-489, 2012.

EL-MASHALEH, Mohammad S.; EDWARD MINCHIN JR, R.; O'BRIEN, W. J. Management of construction firm performance using benchmarking. Journal of Management in Engineering, v. 23 (1), n. January, p. 10-17, 2007.

FILHO, D. B. F.; JÚNIOR, J. A. D. S. Desvendando os mistérios do coeficiente de correlação de Pearson (r). Revista Política Hoje, v. 18, n. 1, p. 115-146, 2009.

FRIMPONG, Y.; OLUWOYE, J.; CRAWFORD, L. Causes of delay 
and cost overruns in construction of groundwater projects in a developing countries; Ghana as a case study. International Journal of Project Management, v. 21, n. 5, p. 321-326, 2003.

FONSECA, D. R. DA et al. Autonomia para gestão estratégica de pessoas no setor público federal: perspectivas de análise e agenda de pesquisa. Revista de Administração Pública, v. 47, n. 6, p. 1451-475, 2013.

FOOLADVAND, M.; YARMOHAMMADIAN, M. H.; SHAHTALEBI, S. The Application Strategic Planning and Balance Scorecard Modelling in Enhance of Higher Education. Procedia - Social and Behavioral Sciences, v. 186, p. 950-954, 2015.

FRANZBLAU, Abraham. A primer of statistics for nonstatisticians. Oxford, England: Harcourt, Brace, Cap. 7, (1958).

GASIK, S. Are public projects different than projects in other sectors? Preliminary results of empirical research. Procedia - Procedia Computer Science, v. 100, p. 399-406, 2016.

GRIN, E. J. Intersetorialidade e transversalidade no modelo de gestão e planejamento governamental no município de Vitória. V Congreso Internacional del CLAD sobre la Reforma del Estado y de la Administración Pública, Santo Domingo, Rep. Dominicana, 24- 27 out., 2000.

HO, A. T. K. PBB in American Local Governments: It's More than a Management Tool. Public Administration Review, v. 71, n. 3, p. 391-401, 2011.

KAHVECI, T. C. et al. Evaluation of public strategic planning models for Turkish Universities. Procedia: Social and Behavioral Sciences, v. 58, p. 138-148, 2012. Doi: 10.1016/j.sbspro.2012.09.987.

KALIBA, C.; MUYA, M.; MUMBA, K. Cost escalation and schedule delays in road construction projects in Zambia. International Journal of Project Management, v. 27, n. 5, p. 522-531, 2009.

KAPLAN, R. S.; NORTON, D. P. The Balanced Scorecard: Measures That Drive Performance. Harvard Business Review, 2005.

KIRSCH, P.; HINE, A.; MAYBURY, T. A model for the implementation of industry-wide knowledge sharing to improve risk management practice. Safety Science, v. 80, p. 66-76, 2015.

LINS, M. E. et al. O uso da Análise Envoltória de Dados (DEA) para avaliação de hospitais universitários Brasileiros.

Ciência e Saúde Coletiva, v. 12, n. 4, p. 985-998, 2007. 
LUU, V. T.; KIM, S. Y.; HUYNH, T. A. Improving project management performance of large contractors using benchmarking approach. Int. J. Project Manage, v. 7, n. 26, 758-769, 2008.

MAGNUSSEN, O. M.; OLSSON, N. O. E. Comparative analysis of cost estimates of major public. International Journal of Project Management, v. 24, n. 4, p. 281-288, 2006.

MOTTA, Paulo Roberto de Mendonça. O estado da arte da gestão pública. Revista de Administração de Empresas, v. 53, n. 1, p. 82-90, 2013.

NASIR, H. et al. Development and implementation of a benchmarking and metrics program for construction performance and productivity improvement. Canadian Journal of Civil Engineering, v. 39, n. 9, p. 957-967, 2012.

PEÑA, C. R.. Um Modelo de Avaliação da Eficiência da Administração Pública Através do Método Análise Envoltória de Dados (DEA). Revista de Administração Contemporânea, V. 12, n. 1, P. 83-106, 2008.

PINHEIRO, J. Indicadores-chave de Desempenho (Key Performance Indicators) aplicados à construção. Dissertação de Mestrado, Instituto Superior TécnicoUniversidade Técnica de Lisboa, 2011.

PUTHAMONT, S.; CHAROENNGAM, C. Strategic project selection in public sector: Construction projects of the Ministry of Defence in Thailand. International Journal Of Project Management, [s.1.], v. 25, n. 2, p.178-188, fev. 2007.

RAMÍREZ, R. R.; ALARCÓN, L. F. C.; KNIGHTS, P. Benchmarking System for Evaluating Management Practices in the Construction Industry. Journal of Management in Engineering, v. 20, n. July, p. 110-117, 2004.

SOIBELMAN, L.; KIM, H.; WU, J. Knowledge Discovery for Project Delay Analysis. Bauingenieur, Springer VDI Verlag, 2005.

SORTE, J. R. L. B. Planejamento e Controle nas Obras da Rede Federal de Educação Profissional, Cientifica e Tecnológica. Revista Especialize On Line - IPOG, v. 1, p. 1-19, 2015.

SOUZA, Rommel Silva. Técnicas gerenciais para o setor de obras públicas: elaboração de indicadores chave de desempenho para o instituto federal catarinense. Dissertação de Mestrado, CENTRO UNIVERSITÁRIO SOCIESC - UNISOCIESC, 2018.

TOOR, S.; OGUNLANA, S. O. Beyond the "iron triangle": 
Stakeholder perception of key performance indicators (KPIs) for large-scale public sector development projects. International Journal of Project Management, v. 28, n. 3, p. 228-236, 2010.

VOELKL, K.; GERBER, S. Using SPSS for Windows: Data analysis and graphics, Springer, New York, 1999.

WATTHANANON, J.; MINGKHWAN, A. Optimizing Knowledge Management using Knowledge Map. Procedia Engineering, v. 32, p. 1169-1177, 2012.

YUN, Sungmin et al. Development of performance metrics for phase-based capital project benchmarking. International Journal of Project Management, v. 34, n. 3, p. 389-402, 2015.

YUSOF, M. N.; HASSAN, A.; BAKAR, A. Knowledge management and growth performance in construction companies: a framework. Procedia Social and Behavioral Sciences, v. 62, p. 128-134, 2012. 